\title{
CENTRALIZERS IN DOMAINS OF GELFAND-KIRILLOV DIMENSION 2
}

\author{
JASON P. BELL AND LANCE W. SMALL
}

\begin{abstract}
Given an affine domain of Gelfand-Kirillov dimension 2 over an algebraically closed field, it is shown that the centralizer of any non-scalar element of this domain is a commutative domain of Gelfand-Kirillov dimension 1 whenever the domain is not polynomial identity. It is shown that the maximal subfields of the quotient division ring of a finitely graded Goldie algebra of GelfandKirillov dimension 2 over a field $F$ all have transcendence degree 1 over $F$. Finally, centralizers of elements in a finitely graded Goldie domain of Gelfand-Kirillov dimension 2 over an algebraically closed field are considered. In this case, it is shown that the centralizer of a non-scalar element is an affine commutative domain of Gelfand-Kirillov dimension 1.
\end{abstract}

\section{Introduction}

In this paper we consider domains of Gelfand-Kirillov dimension 2. This dimension was first considered by Gelfand and Kirillov in 1966 [6]. It is defined as follows. Given a field $F$ and an affine $F$-algebra $A$, we define the Gelfand-Kirillov dimension of $A$ to be

$$
\operatorname{GKdim}(A):=\limsup _{n \rightarrow \infty} \log \left(\operatorname{dim} V^{n}\right) / \log n,
$$

where $V$ is a finite-dimensional subspace of $A$ that generates $A$ as an $F$-algebra. We note that this definition is independent of choice of $V$. In the case that $A$ is not affine, we define the Gelfand-Kirillov dimension of $A$ to be the supremum of the Gelfand-Kirillov dimensions of all affine subalgebras of $A$. Basic facts about Gelfand-Kirillov dimension can be found in [6].

An affine domain of Gelfand-Kirillov dimension 0 is a division ring that is finitedimensional over its center. An affine domain of Gelfand-Kirillov dimension 1 over a field $F$ is a finite module over its center, and hence polynomial identity (see [9]); if, in addition, $F$ is algebraically closed, a routine application of Tsen's theorem shows that this domain is in fact commutative. Domains of Gelfand-Kirillov dimension 2 are not well understood. The second author conjectures that such domains must either be primitive or polynomial identity. In the case that such a domain is a finitely generated $\mathbb{N}$-graded algebra with the property that the homogenous elements of any given degree form a finite-dimensional vector space, it has been shown by Artin and Stafford [2] that this conjecture holds. We consider the centralizers of elements of an affine domain of Gelfand-Kirillov dimension 2. Centralizers of the first Weyl algebra - a domain of Gelfand-Kirillov dimension 2 - were studied as long ago as 1922 by Burchnall and Chaundy [4], and also by Amitsur [1]. Using the work of Artin and Stafford, we are also able to obtain results about the centralizers of

Received 26 March 2003; revised 22 October 2003.

2000 Mathematics Subject Classification 16P90. 
elements of the quotient division algebra of a finitely graded domain of GelfandKirillov dimension 2. We state our main results.

TheOREM 1.1. Let $A$ be a non-polynomial identity affine domain of GelfandKirillov dimension 2 over an algebraically closed field $F$. Then the centralizer of a non-scalar element of $A$ is a commutative domain of Gelfand-Kirillov dimension 1.

In the case where we are dealing with graded rings, we obtain somewhat stronger results. We first give the following definition.

DeFinition 1.2. Let

$$
A=\bigoplus_{n=0}^{\infty} A_{n}
$$

be a finitely generated $\mathbb{N}$-graded $F$-algebra. We say that $A$ is finitely graded if $\operatorname{dim}_{F}\left(A_{n}\right)<\infty$ for all $n \geqslant 0$.

TheOrem 1.3. Let $A$ be a finitely graded non-polynomial identity domain of Gelfand-Kirillov dimension 2 over a field $F$. Then any subfield of $Q(A)$ has transcendence degree at most 1 over $F$.

ThEOREM 1.4. Let $A$ be a finitely graded non-polynomial identity domain of Gelfand-Kirillov dimension 2 over an algebraically closed field $F$. Then the centralizer of a non-scalar element $a \in Q(A)$ is a finitely generated field extension of $F$ of transcendence degree 1.

\section{Algebras of Gelfand-Kirillov dimension 2}

Lemma 2.1. Let $A$ be a non-polynomial identity affine domain of GelfandKirillov dimension 2 over an algebraically closed field $F$, and let $B$ be a polynomial identity subalgebra of $A$. Then $B$ has Gelfand-Kirillov dimension at most 1.

Proof. Suppose that $B$ has Gelfand-Kirillov dimension greater than 1. Then $\operatorname{GKdim}(B)>\operatorname{GKdim}(A)-1$. By a theorem of Borho and Kraft (see $[\mathbf{3}]), Q(A)$ is a finite-dimensional vector space over $Q(B)$. In particular, $Q(A)$ is polynomial identity, since $Q(B)$ is polynomial identity, a contradiction. It follows that $B$ must have Gelfand-Kirillov dimension 1.

TheOREm 2.2. Let $A$ be a non-polynomial identity affine domain of GelfandKirillov dimension 2 over an algebraically closed field $F$. Then the centralizer of a non-scalar element of $A$ is a commutative domain of Gelfand-Kirillov dimension 1.

Proof. Let $a$ be a non-scalar element of $A$. Let $C(a)$ denote the centralizer of $a$ in $A$, and let $B$ be an affine subalgebra of $C(a)$ containing $a$. Notice that the GelfandKirillov dimension of $B$ is at most 2, as it is a subalgebra of $A$. On the other hand, the center of $B$ has Gelfand-Kirillov dimension at least 1 , since it contains $F[a]$. By [10, Corollary 2], $B$ is polynomial identity. By Lemma 2.1, $B$ has Gelfand-Kirillov 
dimension 1. By $[\mathbf{9}], B$ is commutative. Since $B$ is an arbitrary affine subalgebra of $C(a)$, we see that $C(a)$ is a commutative domain of Gelfand-Kirillov dimension 1.

\section{Graded algebras of Gelfand-Kirillov dimension 2}

In this section we prove our main results for graded domains of Gelfand-Kirillov dimension 2. Throughout this section we use arguments involving Krull dimension.

Notation 3.1. Given an algebra $A$ and a right $A$-module $M$, we denote the Krull dimension of $M$ by $\mathcal{K}(M)$.

For basic facts about Krull dimension we refer the reader to [7].

Lemma 3.2. Let $A$ be a prime Noetherian polynomial identity ring that is not primitive. Suppose that $\sigma$ is an automorphism of $A$ such that $A\left[x, x^{-1} ; \sigma\right]$ is simple. Then

$$
\mathcal{K}(A)=\mathcal{K}\left(A\left[x, x^{-1} ; \sigma\right]\right)
$$

Proof. The proof is similar to that of [7, Corollary 6.6.7]. We show that there are no nonzero right $A\left[x, x^{-1} ; \sigma\right]$-modules of finite length over $A$. Suppose that $M$ is a nonzero right $A\left[x, x^{-1} ; \sigma\right]$-module of finite length over $A$. Since $A$ is not primitive, $\operatorname{ann}_{A}(M)$ must contain a finite product of primitive ideals of $A$. Thus $A / \operatorname{ann}_{A}(M)$ is a subdirect product of semisimple Artinian rings. It follows that $\mathcal{K}\left(A / \operatorname{ann}_{A}(M)\right)=$ 0 . Notice that $\operatorname{ann}_{A}(M)$ is stable under $\sigma$, and hence generates a proper nonzero two-sided ideal of $A\left[x, x^{-1} ; \sigma\right]$, contradicting the fact that $A\left[x, x^{-1} ; \sigma\right]$ is simple. The result now follows from [7, Theorem 6.6.10].

We now prove a useful lemma about subfields of quotient division algebras.

Lemma 3.3. Let $A$ be a finitely graded Goldie domain of Gelfand-Kirillov dimension 2. Then the subfields of $Q(A)$ are finitely generated.

Proof. By [2, Theorem 0.1] we know that $A$ has a graded quotient ring

$$
Q_{\mathrm{gr}}(A) \cong D\left[x, x^{-1} ; \sigma\right]
$$

for some division ring $D$ which is a finite module over its center and some automorphism $\sigma$ of $D$. By [8, Theorem 3] the subfields of $Q\left(D\left[x, x^{-1} ; \sigma\right]\right)$ are finitely generated. The result follows.

Proposition 3.4. Let $A$ be a finitely graded non-polynomial identity domain of Gelfand-Kirillov dimension 2 over a field $F$. Then $Q_{\mathrm{gr}}(A)$ is a simple $F$-algebra and $Z\left(Q_{\mathrm{gr}}(A)\right)$ is a finite extension of $F$ consisting of homogeneous elements of degree 0 .

Proof. Note that since $A$ is a finitely generated domain of Gelfand-Kirillov dimension 2, we have

$$
\operatorname{GKdim}(A / I) \leqslant 1
$$


for any nonzero ideal $I$ of $A$. In particular, $A / I$ is polynomial identity, by $[\mathbf{9}]$. It follows that $A / I$ satisfies a multilinear homogeneous polynomial identity, say $p\left(x_{1}, \ldots, x_{d}\right)$. Now since $p$ is multilinear and $A$ is not polynomial identity, there exist homogeneous elements $a_{1}, \ldots, a_{d} \in A$ such that $u:=p\left(a_{1}, \ldots, a_{d}\right) \neq 0$. Thus $u$ is a nonzero homogeneous element of $A$ which is in $I$. It follows that every nonzero ideal of $I$ contains a homogeneous regular element $u$. Thus $Q_{\mathrm{gr}}(A)$ is simple. By $[\mathbf{2}$, Theorem 0.1],

$$
Q_{\mathrm{gr}}(A) \cong D\left[x, x^{-1} ; \sigma\right]
$$

with $D$ finite-dimensional over its center $Z$ and $Z$ a finitely generated field extension of transcendence degree 1 over $F$. Note that if $a \in Q_{\mathrm{gr}}(A)$ is central, then each of the homogeneous parts of $a$ must also be central. Suppose that $b=\alpha x^{i}$ with $\alpha \in D$ and $i \neq 0$ is central. Then since $D$ is a finite module over its center,

$$
D\left[\alpha x^{i},\left(\alpha x^{i}\right)^{-1}\right]=D\left[x^{i}, x^{-i} ; \sigma^{i}\right]
$$

is polynomial identity. Since $D\left[x, x^{-1} ; \sigma\right]$ is a finite module over $D\left[x^{i}, x^{-i} ; \sigma^{i}\right]$, we conclude that $Q_{\mathrm{gr}}(A)$ is polynomial identity, a contradiction. Thus any homogeneous central element of $Q_{\mathrm{gr}}(A)$ must have degree 0. Consequently, $Z\left(Q_{\mathrm{gr}}(A)\right) \subseteq D$. Next, suppose that $\alpha \in D$ is in $Z\left(Q_{\mathrm{gr}}(A)\right)$. Then $F(\alpha)\left[x, x^{-1}\right]$ is commutative. We claim that $\alpha$ is algebraic over $F$. To see this, suppose that this is not the case. Then $[Z: F(\alpha)]<\infty$, and hence $Q_{\mathrm{gr}}(A)$ is a finite module over $F(\alpha)\left[x, x^{-1}\right]$. Since $F(\alpha)\left[x, x^{-1}\right]$ is commutative, we conclude that $Q_{\mathrm{gr}}(A)$ is polynomial identity, which contradicts the fact that $A$ is not polynomial identity. Thus $\alpha$ is algebraic over $F$. It follows that $Z\left(Q_{\mathrm{gr}}(A)\right)$ is an algebraic extension of $F$. By Lemma 3.3, it is also a finitely generated extension. The result follows.

TheOREM 3.5. Let $A$ be a finitely graded non-polynomial identity domain of Gelfand-Kirillov dimension 2 over a field $F$. Then any subfield of $Q(A)$ has transcendence degree at most 1 over $F$.

Proof. By [2, Theorem 0.1], $A$ has a graded quotient

$$
Q_{\text {gr }}(A)=D\left[x, x^{-1} ; \sigma\right],
$$

where $D$ is a division algebra which is finite-dimensional over its center $Z$, with $Z$ a finitely generated extension of $F$ of transcendence degree 1 . Let $K$ be a subfield of $Q_{\mathrm{gr}}(A)$ that is a purely transcendental extension of $F^{\prime}:=Z\left(Q_{\mathrm{gr}}(A)\right) \subseteq D$. Observe that $K \otimes_{F^{\prime}} Q_{\mathrm{gr}}(A) \cong\left(D \otimes_{F^{\prime}} K\right)\left[x, x^{-1} ; \sigma\right]$. Now $D \otimes_{F^{\prime}} K$ is a prime Noetherian algebra. Since it is polynomial identity and not simple, it is not primitive. Note that

$$
Q_{\mathrm{gr}}(A) \otimes_{F^{\prime}} K \cong\left(D \otimes_{F^{\prime}} K\right)\left[x, x^{-1} ; \sigma\right]
$$

is simple, because by Proposition 3.4, $Q_{\mathrm{gr}}(A)$ is a central simple $F^{\prime}$-algebra and $K$ is a simple $F^{\prime}$-algebra. By Lemma 3.2 ,

$$
\mathcal{K}\left(Q_{\text {gr }}(A) \otimes_{F^{\prime}} K\right)=\mathcal{K}\left(D \otimes_{F^{\prime}} K\right) .
$$

Since $D$ is a finite $Z$-module, we have

$$
\mathcal{K}\left(D \otimes_{F^{\prime}} K\right)=\mathcal{K}\left(Z \otimes_{F^{\prime}} K\right)=\min \left\{\operatorname{trdeg}_{F^{\prime}}(Z), \operatorname{trdeg}_{F^{\prime}}(K)\right\},
$$

and so

$$
\mathcal{K}\left(Q_{\mathrm{gr}}(A) \otimes_{F^{\prime}} K\right)=\min \left\{\operatorname{trdeg}_{F^{\prime}}(Z), \operatorname{trdeg}_{F^{\prime}}(K)\right\}
$$


We also have

$$
\operatorname{trdeg}_{F^{\prime}}(K)=\mathcal{K}\left(K \otimes_{F^{\prime}} K\right) .
$$

By [7, Corollary 6.5.3], we have $\mathcal{K}\left(K \otimes_{F^{\prime}} K\right) \leqslant \mathcal{K}\left(Q(A) \otimes_{F^{\prime}} K\right)$. Additionally, by [7, Lemma 6.5.3.ii], we have

$$
\mathcal{K}\left(Q(A) \otimes_{F^{\prime}} K\right) \leqslant \mathcal{K}\left(Q_{\mathrm{gr}}(A) \otimes_{F^{\prime}} K\right)=\min \left(\operatorname{trdeg}_{F^{\prime}}(K), \operatorname{trdeg}_{F^{\prime}}(Z)\right) .
$$

Since $Z$ has transcendence degree at most 1 over $F^{\prime}$, we see that $\operatorname{trdeg}_{F^{\prime}}(K) \leqslant 1$. Since $F^{\prime}$ is a finite extension of $F$, we obtain the desired result.

We are now ready to prove our main result for graded domains of Gelfand-Kirillov dimension 2.

ThEOREM 3.6. Let $A$ be a finitely graded non-polynomial identity domain of Gelfand-Kirillov dimension 2 over an algebraically closed field $F$. Then the centralizer of a non-scalar element $a \in Q(A)$ is a finitely generated field extension of $F$ of transcendence degree 1.

Proof. Observe that $C(a)$ is a division algebra over $F$. Notice also that for any $b \in C(a), a$ and $b$ commute and hence $F(a, b)$ is a field. By Theorem 3.5, $F(a, b)$ has trancendence degree at most 1 over $F$. Thus $b$ is algebraic over $F(a)$, as $F(a)$ has transcendence degree 1 over $F$. It follows that $C(a)$ is a division algebra that is algebraic over $F(a)$. Let $Z$ denote the center of $C(a)$. Then $Z \supseteq F(a)$, and hence $Z$ has transcendence degree at least 1 over $F$. On the other hand, $Z$ is a subfield of $Q(A)$, and hence has transcendence degree at most 1 over $F$. By Lemma 3.3, $Z$ is a finitely generated field extension of $F$. Let $K$ denote a maximal subfield of $C(a)$. Then $K$ has transcendence degree 1 over $F$ and is a finitely generated extension of $F$ by the same lemma. It follows that $K$ is a finitely generated algebraic extension of $Z$, and so $[K: Z]<\infty$. It follows that $C(a)$ is finite-dimensional over $Z$ using the results from $[\mathbf{5}$, p. 165$]$; in fact, $[C(a): Z]=[K: Z]^{2}$. Since $Z$ is a finitely generated extension of transcendence degree 1 of an algebraically closed field, $Z$ is necessarily a $C_{1}$ field. By Tsen's theorem, $C(a)$ is commutative, and hence is a field of transcendence degree 1 over $F$.

Proposition 3.7. Let $B$ be a commutative graded domain of Gelfand-Kirillov dimension 1 over an algebraically closed field $F$. Then there exist positive integers $m_{1}, \ldots, m_{\ell}$ and there exists a homogeneous element $t \in Q_{\mathrm{gr}}(B)$ such that $B=$ $F\left[t^{m_{1}}, \ldots, t^{m_{\ell}}\right]$.

Proof. Let

$$
\mathcal{S}=\{\operatorname{deg}(u) \mid u \in B, u \text { homogeneous }\}
$$

There exists some positive integer $N$ such that every element of $\mathcal{S}$ can be expressed as a nonnegative integer linear combination of elements of $\mathcal{S} \cap\{1,2, \ldots, N\}$. We can choose homogeneous elements $u_{1}, \ldots, u_{\ell} \in B$ such that

$$
\left\{\operatorname{deg}\left(u_{1}\right), \ldots, \operatorname{deg}\left(u_{\ell}\right)\right\}=\mathcal{S} \cap\{1,2, \ldots, N\} .
$$

Let $u \in B$ be homogeneous. Now $R:=F\left[u_{1}, \ldots, u_{\ell}, u\right]$ is a finitely graded commutative domain of Gelfand-Kirillov dimension 1. Since $F$ is algebraically 
closed, it follows that $Q_{\mathrm{gr}}(R) \cong F\left[t, t^{-1}\right]$ where $t$ is homogeneous of degree $\operatorname{gcd}\left(\operatorname{deg}\left(u_{1}\right), \ldots, \operatorname{deg}\left(u_{\ell}\right)\right)$. Since $u_{i}$ is homogeneous and in $F\left[t, t^{-1}\right]$, it follows that $u_{i}=\alpha_{i} t^{m_{i}}$ with $m_{i}=\operatorname{deg}\left(u_{i}\right) / \operatorname{deg}(t)$ and $\alpha_{i} \in F$. Similarly, $u=\alpha t^{m}$ with $m=\operatorname{deg}(u) / \operatorname{deg}(t)$. By construction, $m$ is a nonnegative integer linear combination of $m_{1}, \ldots, m_{\ell}$. It follows that $u \in F\left[u_{1}, \ldots, u_{\ell}\right]=F\left[t^{m_{1}}, \ldots, t^{m_{\ell}}\right]$. Thus

$$
B=F\left[t^{m_{1}}, \ldots, t^{m_{\ell}}\right] \text {. }
$$

Theorem 3.8. Let $A$ be a finitely graded Goldie $F$-algebra with the property that for any homogeneous element of positive degree, $x \in A$, there exist positive integers $m_{1}, \ldots, m_{\ell}$ and a homogeneous element $t \in Q_{\mathrm{gr}}(A)$ of positive degree $d$ such that

$$
C(x)=F\left[t^{m_{1}}, \ldots, t^{m_{\ell}}\right] .
$$

Then the centralizer of any element of $A$ is an affine $F$-algebra.

Proof. Let $a \in A$. Write $a=a_{0}+a_{1}+\ldots+a_{n}$, where $a_{i}$ is homogeneous of degree $i$ and $a_{n} \neq 0$. Without loss of generality, $n>0$. Observe that if $b=$ $b_{0}+\ldots+b_{p} \in C(a)$ with $b_{i}$ homogeneous of degree $i$, then $b_{p} \in C\left(a_{n}\right)$. There exist a homogeneous element $t \in Q_{\mathrm{gr}}(A)$ of degree $d$ and positive integers $m_{1}, \ldots, m_{\ell}$ such that $C\left(a_{n}\right)=F\left[t^{m_{1}}, \ldots, t^{m_{\ell}}\right]$. It follows that if $b=b_{0}+\ldots+b_{p} \in C(a)$, then $b_{p}=\alpha t^{m}$, with $\alpha \in F$ and $m \in \mathbb{N} m_{1}+\ldots+\mathbb{N} m_{\ell}$. Let

$$
\mathcal{S}=\{\operatorname{deg}(b) \mid b \in C(a), b \neq 0\} .
$$

Let $d^{\prime}$ be the greatest common divisor of $\mathcal{S}$. Then there exists an integer $N$ such that all integers larger than $N$ can be expressed as a nonnegative integer linear combination of elements of $\mathcal{S}$. Choose elements $r_{1}, \ldots, r_{\ell} \in C(a)$ such that

$$
\left\{\operatorname{deg}\left(r_{1}\right), \ldots, \operatorname{deg}\left(r_{\ell}\right)\right\}=\mathcal{S} \cap\{0,1, \ldots, N\} .
$$

Since the leading homogeneous part of $r_{i}$ is in $C\left(a_{n}\right)$, we can multiply by appropriate scalars so that the leading homogeneous part of $r_{i}$ is $t^{m_{i}}$ for some positive integer $m_{i}$. We claim that $C(a)=F\left[r_{1}, \ldots, r_{\ell}\right]$. To see this, suppose this is not the case. Choose $b=b_{0}+\ldots+b_{p} \in C(a) \backslash F\left[r_{1}, \ldots, r_{\ell}\right]$ with $p$ minimal. Again, we may assume that $b_{p}=t^{p / d}$. By assumption, $p / d=i_{1} m_{1}+\ldots+i_{\ell} m_{\ell}$ for some nonnegative integers $i_{1}, \ldots, i_{\ell}$. Observe that both $b$ and $b^{\prime}:=r_{1}^{i_{1}} \ldots r_{\ell}^{i_{\ell}}$ have degree $p$ and both have the same homogeneous part of degree $p$, namely $t^{p / d}$. Thus $b-b^{\prime} \in C(a)$ has degree at most $p-1$. By the minimality of $\operatorname{deg}(b)$, we see that $b-b^{\prime} \in F\left[r_{1}, \ldots, r_{\ell}\right]$, contradicting the fact that $b \notin F\left[r_{1}, \ldots, r_{\ell}\right]$.

Corollary 3.9. Let $A$ be a finitely graded non-polynomial identity Goldie domain of Gelfand-Kirillov dimension 2 over an algebraically closed field $F$. Then the centralizer of a non-scalar element is an affine commutative domain of GelfandKirillov dimension 1.

Proof. By Theorem 2.2, the centralizer of a non-scalar element $b \in A$ is a commutative domain of Gelfand-Kirillov dimension 1. Hence by Proposition 3.7 and Theorem 3.8, the centralizer of $a$ is affine.

We make the conjecture that the same result holds in the ungraded case. 
Conjecture 3.10. Let $A$ be an affine Noetherian non-polynomial identity domain of Gelfand-Kirillov dimension 2 over an algebraically closed field. Then the centralizer of a non-scalar element is an affine domain.

Corollary 3.11. Let $A$ be an affine domain of Gelfand-Kirillov dimension 2 with a non-polynomial identity domain for an associated graded ring. Then the centralizer of a non-scalar element is an affine commutative domain of GelfandKirillov dimension 1.

Proof. The same argument used in Theorem 3.8 and Corollary 3.9 gives the result.

Acknowledgments. We thank the referee for many helpful comments and suggestions, which greatly improved this paper.

\section{References}

1. S. A. Amitsur, 'Commutative linear differential operators', Pacific J. Math. 8 (1958) 1-10.

2. M. Artin and J. T. StAfFord, 'Noncommutative graded domains with quadratic growth', Invent. Math. 122 (1995) 231-276.

3. W. Borho and H. Kraft, 'Über die Gelfand-Kirillov-Dimension', Math. Ann. 220 (1976) $1-24$.

4. J. L. Burchnall and T. W. Chaundy, 'Commutative ordinary differential operations', Proc. London Math. Soc. (2) 21 (1922) 420-440.

5. N. Jacobson, Structure of rings, Amer. Math. Soc. Colloq. Publ. 37 (Amer. Math. Soc., Providence, RI, 1964).

6. G. R. Krause and T. H. Lenagan, Growth of algebras and Gelfand-Kirillov dimension, revised edn, Graduate Studies in Mathematics 22 (Amer. Math. Soc., Providence, RI, 2000).

7. J. C. MCConnell and J. C. Robson, Noncommutative Noetherian rings (Wiley, New York, 1987).

8. R. Resco, L. W. Small and A. Wadsworth, 'Tensor products of division rings and finite generation of subfields', Proc. Amer. Math. Soc. 77 (1979) 7-10.

9. L. W. Small and R. B. Warfield JR., 'Prime affine algebras of Gelfand-Kirillov dimension one', J. Algebra 91 (1984) 386-389.

10. S. P. Sмith and J. J. Zhang, 'A remark on Gelfand-Kirillov dimension', Proc. Amer. Math. Soc. 126 (1998) 349-352.

\author{
Jason P. Bell \\ Department of Mathematics \\ University of Michigan \\ East Hall \\ 525 East University Ave. \\ Ann Arbor \\ MI 48109-1109 \\ USA
}

belljp@math.lsa.umich.edu

\author{
Lance W. Small \\ Department of Mathematics \\ University of California San Diego \\ La Jolla \\ CA 92093-0112 \\ USA \\ lwsmall@ucsd.edu
}

